\title{
INTERVENCIÓN EDUCATIVA PARA MODIFICAR EL APORTE DE HIERRO ABSORBIBLE EN MUJERES NO EMBARAZADAS
}

\section{EDUCATIONAL INTERVENTION TO MODIFY THE LEVELS OF ABSORBED IRON IN NON PREGNANT WOMEN}

\author{
Emma Edith Biolley H., Luis Humberto Bustos M., Carolina Alejandra Daroch H., \\ Marion Thamara Guerrero W., Dilia Stefany Morales F., Natalia Soledad Ramos N. \\ Departamento de Salud Pública. Facultad de Medicina, Carrera Nutrición y Dietética \\ Universidad de La Frontera, Temuco, Chile.
}

\begin{abstract}
Biochemical tests (hemoglobin and ferritin) and dietary surveys were carried out in this study to determine changes in absorbable iron intake and serum levels of iron, before and after an educational intervention aimed to promote food dietary iron bioavailability in 49 non pregnant women between 18 and 25 year of age students from La Frontera University who were enrolled in this institution in 2009. Favorable changes were detected in vitamin $C$ intake which increased from 67 to $125 \mathrm{mg}(p<0.001)$, and hemoglobin from of 13.2 to $13.4 \mathrm{~g} / \mathrm{dl}(p<0.0065)$. A significant a decrease in factors on the non-heme iron absorption depressants from $100 \%$ to $6.1 \%(p=0.008)$ was observed. Important qualitative chances on the adequate selection and combination of types of food were identified which contributes to improve iron absorption.
\end{abstract}

Key words: anemia, iron deficiency, absorbable iron, iron stores, serum ferritin.

Este trabajo fue recibido el 1 de Diciembre de 2010, aceptado con modificaciones el 3 de Enero de 2011 y aceptado para ser publicado el 30 de Diciembre de 2011.

\section{INTRODUCCIÓN}

Uno de los problemas de salud pública relevantes a nivel mundial, es la alta prevalencia de anemia y de deficiencia de hierro, problemática que adquiere mayor importancia la que se presenta principalmente en grupos de alta vulnerabilidad como lo son niños menores de 2 años, mujeres en edad fértil y embarazadas.

Debido a la alta prevalencia de anemia por déficit de hierro, se han implementado diversas estrategias a nivel nacional, como los programas que contribuyen a solucionar directamente el problema; fortificación de algunos alimentos con hierro y los programas de suplementación con hierro, e indirectamente el programa mundialmente conocido " 5 al día"; cuyo propósito es aumentar el consumo de frutas y verduras en la población; al ser alimentos con alto contenido de vitamina $\mathrm{C}$, contribuyen a aumentar la absorción de hierro no hem entre otros beneficios.

En este trabajo también se consideró relevante mencionar la influencia favorable sobre la prevención de anemia de los programas que buscan disminuir el consumo de alimentos con bajo contenido de micronutrientes (con alto contenido de grasas saturadas y carbohidratos refinados) los cuales limitan el consumo de vitaminas y micronutrientes como el hierro.

El consumo inadecuado de nutrientes a nivel poblacional originó el concepto de "nutrientes críticos" entendiéndose por ellos los nutrientes que presentan en forma habitual una ingesta inadecuada. Tradicionalmente entre los nutrientes críticos deficitarios, se han identificado el hierro, vitaminas como C, A, E, y ácido fólico, entre otros.

Este estudio se orientó a dos nutrientes que presentan una baja cobertura de acuerdo a los hábitos alimentarios de nuestra población; hierro y vitamina $C$.

El aporte de hierro dietético se obtiene en gran proporción a partir de hierro no hem, presente principalmente en cereales, leguminosas y vegetales; cabe destacar que la absorción de éste hierro es influenciada por otros componentes dietéticos que favorecen o disminuyen este 
proceso. A diferencia del hierro hem (hemínico), cuya absorción no está influenciada por la dieta (a excepción del calcio y la lactosa) cuya absorción se estima en alrededor del $23 \%$ (1).

La baja biodisponibilidad de hierro de la dieta occidental en general, ha contribuido a lo largo del tiempo a mantener una alta la prevalencia de anemia; lo cual también ocurre en nuestro país; no obstante esta situación es contrarrestada por el consumo excesivo de pan fortificado con hierro de nuestra población pero agudizado por el bajo consumo de factores favorecedores de la absorción de éste mineral, conducta que contribuye a generar dietas de baja biodisponibilidad.

De acuerdo a los antecedente analizados el objetivo de este estudio fue orientar a través de una intervención educativa alimentaria, en relación a la selección y combinación de alimentos que favorezcan la absorción de hierro no hem, contribuyendo de esta forma a prevenir la anemia y deficiencia de hierro en mujeres entre 18 y 25 años de edad, de la Universidad de La Frontera, durante el segundo semestre del año 2009.

\section{SUJETOS Y MÉTODO}

Estudio pre-experimental de tipo antes y después con grupo único. Para estimar la muestra del estudio, se consideró la población de 3215 mujeres entre 18 y 25 años de edad, matriculadas en la Universidad de La Frontera, durante el año 2009. Se consideró la referencia de consumo promedio de hierro dietético esperado de $11.5 \mathrm{mg} /$ día y desviación estándar (DS) de 4.6 (1) y un promedio mínimo de consumo de $9.5 \mathrm{mg} /$ día, con un nivel de significación del 5\%, confianza un $95 \%$ y poder un $80 \%$. A la muestra de 48 estudiantes se agregó un $20 \%$ de sobremuestra por posibles deserciones. La selección de las estudiantes se realizó con un muestreo aleatorio estratificado con asignación proporcional en las facultades pertenecientes a la Universidad de La Frontera. Se excluyeron las que presentaron anemia y las que no asistieron regularmente a las sesiones educativas, obteniéndose una muestra final de 49 alumnas.

El estudio fue aprobado por el Comité de Ética de la Facultad de Medicina de la Universidad de La Frontera.

Antes de la intervención se aplicaron dos encuestas alimentarias por recordatorio de 24 horas, durante dos días no consecutivos (día hábil y no hábil). Simultáneamente se realizaron los exámenes bioquímicos; ferritina sérica para detectar deficiencia de hierro $<15 \mathrm{ng} / \mathrm{ml}$ y $<20 \mathrm{ng} / \mathrm{ml}$ definir depósitos de hierro insuficientes $(2,3$ 4)) a través del método quimioluminiscencia activada y hemoglobina $(\mathrm{Hb})$ (indicador de anemia $\mathrm{Hb}<12 \mathrm{mg} / \mathrm{dl}$ (5) con el método de cianmetahemoglobina.

Posteriormente se realizó la intervención educativa
"Anemia tú enemigo silencioso". La cual contempló cinco sesiones educativas grupales, con el fin de orientar hacia un mayor consumo de vitamina $\mathrm{C}$ para aumentar el hierro absorbible en la dieta habitual de las participantes.

Finalizadas las sesiones educativas se aplicaron nuevamente dos encuestas alimentarias y de 2 meses post intervención los exámenes ferritina sérica y hemoglobina. Se compararon los resultados pre y post intervención para evaluar posibles modificaciones en la alimentación de las participantes.

Los métodos estadísticos para el análisis descriptivo fueron; promedio, desviación estándar (SD), mínimo, máximo, porcentaje. Para el análisis inferencial se utilizaron las pruebas de signos y rangos de Wilcoxon (6).

\section{RESULTADOS}

En una muestra de 58 mujeres, pre intervención se detectó un $5,1 \%$ de anemia y $22,5 \%$ con deficiencia de hierro, excluyéndose del estudio a las alumnas que presentaron anemia y a las que no asistieron regularmente a las sesiones educativas, quedando una muestra final de 49 alumnas, en las cuales se realizó la intervención educativa, presentaron un 20,4\% de deficiencia de hierro.

Dada la relación existente entre capacidad adquisitiva y el acceso a alimentos ricos en hierro hem, se utilizó la variable previsión para obtener información indirecta del nivel socioeconómico de las participantes, encontrándose que el $75,5 \%$ era tipo B.

Considerando que los fumadores tienen mayores pérdidas de vitamina $\mathrm{C}$, se investigó sobre el hábito tabáquico de las participantes. Se pesquisaron 10 alumnas fumadoras, con un consumo promedio diario de 3,3 cigarrillos aproximadamente.

También se reunieron antecedentes sobre el método anticonceptivo utilizado por las participantes, ya que según se trate de anticonceptivo oral o dispositivo intrauterino, se pueden observar diferentes pérdidas de hierro.

La mayoría de las mujeres presentaron ciclos menstruales regulares (cada 30 días), con una duración promedio de 5 días. Alrededor del $57 \%$ de las participantes reconocieron presentar un flujo menstrual abundante.

Considerando la influencia del tipo de método anticonceptivo sobre las pérdidas de hierro, 13 mujeres señalaron utilizar algún método anticonceptivo, el más frecuente fue el anticonceptivo oral $(n=11)$, método que se caracteriza por menores pérdidas hemáticas en relación al dispositivo intrauterino (DIU) (7)

$\mathrm{El}$ aporte promedio de vitamina $\mathrm{C}$ pre intervención fue de $67 \mathrm{mg}$ /día, con una adecuación de aproximadamente un 80\% (DRI 1997-2001) (8) aporte que aumentó a $115 \mathrm{mg} /$ día post intervención, obteniéndose una adecuación de $136 \%$. Se observa que el consumo de este 
nutriente disminuyó en 7 mujeres y aumento en 41 siendo estos cambios estadísticamente significativos $(\mathrm{p}<0,001)$.

El consumo promedio de carne pre intervención fue de $109 \mathrm{~g}$ diarios, aumentando a $114 \mathrm{~g}$ post intervención, observándose que alrededor del $47 \%$ aumentó el consumo de este producto, cambio no significativa ( $\mathrm{p}=$ 0,7578 ). Cabe destacar que el tipo de carne que predominó en la alimentación de las alumnas, fue el consumo de carnes rojas.

El aporte de hierro total se mantuvo durante el periodo de observación, promedio aproximado de 13 $\operatorname{mg}(\mathrm{p}=0,8152)$.

Es importante señalar que el principal mecanismo que se aplicó para aumentar la absorción de hierro no hem en este estudio, fue la promoción de un mayor consumo y mejor distribución de alimentos con alto contenido de vitamina $\mathrm{C}$ durante los horarios de comida, debido a que es económicamente más accesible que los cárneos, aportando además; vitaminas, fibra y antioxidantes, asociados a la prevención de enfermedades crónicas no transmisibles, siendo el consumo muy similar pre y post intervención $(\mathrm{p}=0,8384)$. Los resultados de las encuestas por recordatorio de 24 horas, señalan que el aporte calórico y de hierro total provenía principalmente de alimentos ricos en hidratos de carbono como pan y sus derivados (situación característica de la dieta occidental), a nivel nacional a base de harina fortificada con hierro (Reglamento Sanitario de los Alimentos en Chile, Mayo de 2008, título XV- de los farináceos, Párrafo II- de la harinas, Art.350). (9) agregar esta referencia reglamento sanitario.

En relación a la participación del hierro hem en la alimentación de las alumnas, inicialmente se obtuvo un consumo promedio de $0,99 \mathrm{mg} /$ día, aumentando posterior a la intervención a 1,18 mg/día, observándose un importante incremento en el consumo de este tipo de hierro; este cambio no fue estadísticamente significativo $(\mathrm{p}=0,1255)$.

Como es sabido en la evaluación de la calidad del hierro de una dieta, más que conseguir una alta cobertura de hierro total, se debe asegurar un adecuado aporte de hierro absorbible, el que condiciona la utilización de hierro en el organismo.

Se detectaron diferencias pre y post intervención en el promedio de hierro absorbible, aumentando de $0,83 \mathrm{mg} /$ día a $0,90 \mathrm{mg} /$ día posterior a la intervención. Si bien no hubo cambios significativos ( $\mathrm{p}=0,1183)$, sí se presentaron cambios biológicamente importantes en 31 estudiantes, ya que el aumento del aporte de hierro absorbible es la base de la prevención de la deficiencia de hierro y anemia.

Para favorecer el aporte de hierro absorbible de la dieta, se considera un adecuado aporte de hierro hem, además de hierro no hem y la interacción entre este último y factores dietéticos promotores de su absorción, como el consumo de vitamina $\mathrm{C}$ y carne.

La absorción de hierro no hem es negativamente afectada por el consumo simultáneo de factores depresores como; café, té, mate, entre otros. A fin de conocer la potencial influencia de estos depresores se analizó la participación de ellos en la alimentación de las alumnas, comprobándose una marcada disminución post intervención de $100 \%$ a $6,1 \%(p=0,008)$ (tabla 1$)$

Para evaluar el comportamiento de los indicadores bioquímicos se trabajó con hemoglobina para diagnosticar anemia y ferritina sérica para detectar depósitos de hierro insuficientes.

Los niveles promedio de hemoglobina aumentaron

\section{TABLA 1}

Aporte de vitamina $\mathrm{C}$, carne, hierro hem, no hem, y hierro total pre y post intervención

\begin{tabular}{|lcccccccc|}
\hline & \multicolumn{3}{c}{ Pre-interv. } & \multicolumn{3}{c|}{ Post-interv } & \multicolumn{3}{c|}{ Modificaciones } \\
Variable & $\mathbf{X}$ & $\mathbf{D E}$ & $\mathbf{X}$ & $\mathbf{D E}$ & Disminuyen & Aumentan & Cero & $\mathbf{p}$ \\
\hline Vitamina C & 67 & 55,07 & 115 & 69,26 & 7 & 41 & 1 & $<0,0001$ \\
Carne & 109 & 57,91 & 114 & 59,70 & 23 & 26 & 0 & 0,7578 \\
Hierro total & 13,8 & 5,19 & 13,6 & 3,94 & 23 & 26 & 0 & 0,8152 \\
Hierro no hem & 13 & 5,09 & 12 & 3,79 & 23 & 26 & 0 & 0,8384 \\
Hierro hem & 0,99 & 0,52 & 1,18 & 0,70 & 17 & 32 & 0 & 0,1255 \\
Hierro Absorbible & 0,83 & 0,35 & 0,90 & 0,32 & 17 & 31 & 1 & 0,1183 \\
\hline Prueba de signos y rangos de Wilcoxon, X=Media, DE =Desviación estándar & & & & & \\
\hline
\end{tabular}


en el $63 \%$ de las alumnas $(n=31)$, con un promedio de $13,2 \mathrm{~g} / \mathrm{dl}$ a 13,4 g/dl post intervención, diferencia estadísticamente significativa $(\mathrm{p}=0,0065)$.

Situación distinta ocurrió con los niveles de ferritina sérica promedio, pre y post intervención, variando de $30,3 \mathrm{ng} / \mathrm{ml}$ a $29,4 \mathrm{ng} / \mathrm{ml}$, no detectándose cambios en la concentración de este indicador $(\mathrm{p}=0,5775)$.

\section{DISCUSIÓN}

Previo a la intervención educativa, se detectó un $5,1 \%$ de anemia, destacando su similitud con los resultados reportados a nivel nacional $(5,7 \%)$ por el Ministerio de Salud en mujeres no embarazadas entre 17 y 24 años, el año 2003 (10).

Se identificó un 30,5\% de alumnas con deficiencia de hierro o depósitos insuficientes, similar al 27,03\% descrito por Ortega y col. el año 2009 en MaracaiboVenezuela (11).

En relación a la vitamina $\mathrm{C}$, pre intervención se detectó un aporte promedio levemente bajo las recomendaciones (65mg/día) (DRI 1997-2001) situación similar a la reportada por Andrews A y col. el año 2007 (12), (68 mg/día), la cual cambió notablemente post intervención, posiblemente por la acción educativa para aumentar su consumo.

El aporte de hierro total antes y después de la intervención fue estadísticamente similar, con un aporte promedio de $12,76 \mathrm{mg} /$ día. Cifra mayor al descrito por Rebolledo A. y col. el año 2005 (13) (11,5mg/día) en un grupo de 54 mujeres no embarazadas, aparentemente sanas de la Región Metropolitana.

Como es sabido, la mayor parte del hierro de la dieta occidental, corresponde a hierro no hem, similar a lo detectada en la alimentación de las alumnas, por la ingesta de alimentos elaborados con harina de trigo fortificada con hierro. Nuestro país se destaca como uno de los mayores consumidores de pan en el mundo (pro- medio $180 \mathrm{~g} /$ día). Las variaciones del aporte de hierro no hem tuvieron un promedio de $12,77 \mathrm{mg} /$ día y $12,4 \mathrm{mg} /$ día pre y post intervención respectivamente, diferencia estadísticamente no significativa, que puede reflejar el hábito de consumo de pan en nuestro país.

El hierro hem en la dieta de las alumnas, aumentó levemente post intervención, con un aporte promedio de $0,99 \mathrm{mg} /$ día a $1,18 \mathrm{mg} /$ día, diferencia no significativa, pero de gran relevancia biológica, debido al doble rol que cumple el hierro hem; su mayor absorción y capacidad de mejorar la absorción del hierro no hem (3\% a 8\%).

El resultado del estudio señala la conveniencia de incluir un adecuado aporte y correcta distribución de los promotores de la absorción de hierro no hem como vitamina $\mathrm{C}$ y carne durante los diferentes horarios de comida, para favorecer la absorción de hierro de consumo habitual.

De acuerdo a los niveles séricos de hierro; el promedio de hemoglobina al inicio de la intervención fue 13,17 g/dl, similar al descrito por Meertens L y col. el año 2002 (9) (12,9 g/dl), en un grupo de 60 mujeres en edad fértil no embarazadas y aparentemente sanas.

Respecto a las reservas de hierro, el promedio de ferritina pre intervención fue de 30,27 ng/ml, similar al $30,7 \mathrm{ng} / \mathrm{ml}$ reportado por Meertens $\mathrm{L}$ y col el año 2002 (9).

El periodo de observación de dos meses limitó la expresión del efecto de los cambios logrados en la conducta alimentaria de las estudiantes, un mayor tiempo de observación permitiría acentuar los cambios detectados.

\section{CONCLUSIONES}

Al inicio de la intervención se detectó un porcentaje de anemia de $5,1 \%$, mientras que la deficiencia de hierro alcanzó un 30,5\%. Esta situación demuestra que esta problemática aún constituye un problema de Salud Pública en nuestro país.

\section{TABLA 2}

\section{Niveles de hemoglobina y ferritina sérica en 49 mujeres}

\begin{tabular}{|c|c|c|c|c|c|c|c|c|}
\hline \multirow[b]{2}{*}{ Variable } & \multicolumn{2}{|c|}{ Pre-interv. } & \multicolumn{2}{|c|}{ Post-interv } & \multicolumn{4}{|c|}{ Modificaciones } \\
\hline & $\mathbf{X}$ & DE & $\mathbf{X}$ & DE & Disminuyen & Aumentan & Cero & $\mathbf{p}$ \\
\hline $\mathrm{Hb}^{*}$ & 13,2 & 0,69 & 13,4 & 0,66 & 15 & 31 & 3 & 0,0065 \\
\hline Ferritina** & 30,3 & 22,59 & 29,4 & 22,04 & 24 & 24 & 1 & 0,5775 \\
\hline
\end{tabular}


El aporte dietético de vitamina $\mathrm{C}$, pre intervención fue de $67 \mathrm{mg}$ /día, bajo las recomendaciones (DRI, 1997-2001) el que incrementó post intervención a 115 $\mathrm{mg} /$ día, atribuible principalmente a los contenidos tratados.

El aporte de hierro total; no cubrió las recomendaciones (DRI, 1997-2001), pre y post intervención. Cabe destacar que, debido a la tendencia del elevado consumo de pan y otros productos elaborados con harina de trigo en Chile, es que se han implementado programas de enriquecimiento de harina de trigo con este mineral, para favorecer de ésta forma que el beneficio llegará a los distintos grupos poblacionales.

En relación a la cobertura promedio de hierro absorbible en las dietas analizadas, se detectaron leves variaciones positivas (no significativas), pre $(0,83 \mathrm{mg})$ y post intervención $(0,90 \mathrm{mg})$.

Al inicio de la intervención los factores depresores de la absorción de hierro, como los son el consumo de té, café, mate, bebidas gaseosas, cacao, entre otros, eran consumidos en forma habitual por el $100 \%$ de las alumnas, post intervención este consumo disminuyó a sólo un $6,1 \%(\mathrm{p}=0,008)$.

La concentración de hemoglobina, aumentó significativamente post intervención ( $\mathrm{p}<0,001)$. Esta variación podría ser en parte resultado de la disminución de los factores depresores de la absorción de hierro y aumento de los promotores de la misma, en la alimentación de las alumnas post intervención.

La ferritina sérica no tuvo variaciones significativas $(\mathrm{p}=0,57)$ post intervención, presentando un comportamiento asimétrico, reflejando la prioridad biológica de aumentar hemoglobina, antes de aumentar los depósitos de hierro.

\section{RESUMEN}

Se realizó un estudio en 49 alumnas entre 18 y 25 años de edad, de la Universidad de La Frontera, matriculadas el año 2009 con exámenes bioquímicos (hemoglobina y ferritina) y encuestas alimentarias, para determinar modificaciones en el aporte de hierro absorbible y en los niveles séricos de hierro, antes y después a una intervención educativa alimentaria enfocada a favorecer la biodisponibilidad de hierro dietario. Después de la intervención se detectaron cambios favorables ya que el aporte de vitamina $\mathrm{C}$ aumentó de 67 a $125 \mathrm{mg}(\mathrm{p}<0,001)$, la concentración de hemoglobina varió de 13.2 a $13.4 \mathrm{~g} / \mathrm{dl}(\mathrm{p}<0,001)$, y disminuyeron los depresores de absorción de hierro dietético de $100 \%$ a $6,1 \%(p=0,008)$. Se identificaron importantes cambios cualitativos respecto a la correcta selección y combinación de alimentos contribuyendo así a disminuir la deficiencia de hierro en las participantes. Se aportan antecedentes actualizados sobre la calidad de hierro en la dieta y deficiencia de hierro en mujeres en edad fértil.

Palabras clave: anemia, deficiencia de hierro, hierro absorbible, depósitos de hierro, ferritina sérica.

Dirigir la correspondencia a:

Profesora

Emma Edith Biolley H.

Magíster en Nutrición

Docente de la Carrera de Nutrición y Dietética

Departamento de Salud Pública,

Facultad de Medicina

Universidad de La Frontera, Temuco

Fono: 45-484929

Fax: 45-214071

E-mail: ebiolley5@gmail.com

\section{BIBLIOGRAFÍA}

1. Monsen E.R.L.Halberg, M Layrise, D.M.,hegsted, J.D. Cook, W. Mertz C.A Finch. Estimation of available dietary iron. Am J Clin Nutr 1978, 86: 1366-72.

2. World Health Organization/United Nations University/ UNICEF Iron deficiency anemia, assessment, prevention and control: a guide for programme managers. Geneva: WHO, 2001.

3. Centers for Disease Control and prevention. Criteria for anemia in children and childbearing aged women. MMWR Morb Mortal Wkly Rep 1989; 38: 400-4.

4. WHO, CDC. Assessing the iron status of populations: including literatura reviews:report of Joint World health Organization/Centers for Disease Control and Prevention Technical Consultation on the Assessment of Iron Status al the Population Level, Geneva, Swizerlan, 6-8 April 2004. 2nd ed. W, Geneva, Swizerland, 2007.

5. United Nations, Children's Fund, United Nations University, World Health Organization. Iron Deficiency Anaemia Assessment, Prevention, and Control. A guide or programme managers. World Health Organization, 2001.

6. Vieytes, Rut. Metodología de la investigación en organizaciones, mercado y sociedad. $1^{\mathrm{a}}$ ed Argentina, B.A.: De las Ciencias, 2004. pp.370, 584.

7. Meertens L. Solano L Sánchez A. Hemoglobina, ferritina y zinc sérico de mujeres en edad reproductiva: sui asociación con el uso de anticonceptivos. An Venez Nutr 2002; 15(1): 5-10.

8. DRI 1997-2001 Dietary References Intake-Food and Nutrition Board. Institute of Medicine, National Academy of Sciences, USA. 
9. Reglamento Sanitario de los Alimentos en Chile, título XV-de los farináceos, Párrafo II- de la harinas, Art.350, Mayo de 2008.

10. Encuesta Nacional de Salud. Ministerio de Salud, Chile. http:/ epi. Minsal.cl 2003.

11. Ortega P. Leal Montiel J Amaya D Chávez C Anemia y depleción de las reservas de hierro en adolescentes del sexo femenino no embarazadas Rev Chil Nutr 2009:36 (2):111-9.
12. Andrews A. Acosta A. Salas J. Ingesta de vitaminas hidrosolubles en mujeres, estudiantes de medicina de la Universidad de Chile versus requerimientos diarios. Rev ANACEM 2007; vol.1.

13. Rebolledo A. Vásquez M. Del Canto B. Ruz M. Evaluación de la calidad y suficiencia de la alimentación de un grupo de mujeres de la Región Metropolitana de Chile. Rev Chil Nutr 2005; 32(2): 118-25. 